\title{
Glutamine and cancer: cell biology, physiology, and clinical opportunities
}

\author{
Christopher T. Hensley, ${ }^{1}$ Ajla T. Wasti, ${ }^{1,2}$ and Ralph J. DeBerardinis ${ }^{1,2}$
}

${ }^{1}$ Children's Medical Center Research Institute and 2Department of Pediatrics, University of Texas Southwestern Medical Center, Dallas, Texas, USA.

\begin{abstract}
Glutamine is an abundant and versatile nutrient that participates in energy formation, redox homeostasis, macromolecular synthesis, and signaling in cancer cells. These characteristics make glutamine metabolism an appealing target for new clinical strategies to detect, monitor, and treat cancer. Here we review the metabolic functions of glutamine as a super nutrient and the surprising roles of glutamine in supporting the biological hallmarks of malignancy. We also review recent efforts in imaging and therapeutics to exploit tumor cell glutamine dependence, discuss some of the challenges in this arena, and suggest a disease-focused paradigm to deploy these emerging approaches.
\end{abstract}

\section{Introduction}

It has been nearly a century since the discovery that tumors display metabolic activities that distinguish them from differentiated, non-proliferating tissues and presumably contribute to their supraphysiological survival and growth (1). Interest in cancer metabolism was boosted by discoveries that oncogenes and tumor suppressors could regulate nutrient metabolism, and that mutations in some metabolic enzymes participate in the development of malignancy $(2,3)$. The persistent appeal of cancer metabolism as a line of investigation lies both in its ability to uncover fundamental aspects of malignancy and in the translational potential of exploiting cancer metabolism to improve the way we diagnose, monitor, and treat cancer. Furthermore, an improved understanding of how altered metabolism contributes to cancer has a high potential for synergy with translational efforts. For example, the demonstration that asparagine is a conditionally essential nutrient in rapidly growing cancer cells paved the way for L-asparaginase therapy in leukemia. Additionally, the avidity of some tumors for glucose uptake led to the development of ${ }^{18}$ fluoro-2-deoxyglucose imaging by PET; this in turn stimulated hundreds of studies on the biological underpinnings of tumor glucose metabolism.

There continue to be large gaps in understanding which metabolic pathways are altered in cancer, whether these alterations benefit the tumor in a substantive way, and how this information could be used in clinical oncology. In this Review, we consider glutamine, a highly versatile nutrient whose metabolism has implications for tumor cell biology, metabolic imaging, and perhaps novel therapeutics.

\section{Glutamine in intermediary metabolism}

Glutamine metabolism has been reviewed extensively and is briefly outlined here $(4,5)$. The importance of glutamine as a nutrient in cancer derives from its abilities to donate its nitrogen and carbon into an array of growth-promoting pathways (Figure 1). At concentrations of $0.6-0.9 \mathrm{mmol} / \mathrm{l}$, glutamine is the most abundant amino acid in plasma (6). Although most tissues can synthesize glutamine, during periods of rapid growth or other stresses, demand outpaces supply, and glutamine becomes conditionally essential (7).

Authorship note: Christopher T. Hensley and Ajla T. Wasti contributed equally to this work.

Conflict of interest: Ralph J. DeBerardinis is on the scientific advisory board of Peloton Therapeutics.

Citation for this article: J Clin Invest. 2013;123(9):3678-3684. doi:10.1172/JCI69600.
This requirement for glutamine is particularly true in cancer cells, many of which display oncogene-dependent addictions to glutamine in culture (8). Glutamine catabolism begins with its conversion to glutamate in reactions that either donate the amide nitrogen to biosynthetic pathways or release it as ammonia. The latter reactions are catalyzed by the glutaminases (GLSs), of which several isozymes are encoded by human genes GLS and GLS2 (9). Classical studies revealed that GLS isozymes, particularly those encoded by GLS, are expressed in experimental tumors in rats and mice, where their enzyme activity correlates with growth rate and malignancy. Silencing GLS expression or inhibiting GLS activity is sufficient to delay tumor growth in a number of models (10-13). The role of GLS2 in cancer appears to be context specific and regulated by factors that are still incompletely characterized. In some tissues, GLS2 is a p53 target gene and seems to function in tumor suppression (14). On the other hand, GLS2 expression is enhanced in some neuroblastomas, where it contributes to cell survival (15). These observations, coupled with the demonstration that c-Myc stimulates GLS expression $(12,16)$, position at least some of the GLS isozymes as pro-oncogenic.

Glutamate, the product of the GLS reaction, is a precursor of glutathione, the major cellular antioxidant. It is also the source of amino groups for nonessential amino acids like alanine, aspartate, serine, and glycine, all of which are required for macromolecular synthesis. In glutamine-consuming cells, glutamate is also the major source of $\alpha$-ketoglutarate, a TCA cycle intermediate and substrate for dioxygenases that modify proteins and DNA. These dioxygenases include prolyl hydroxylases, histone demethylases, and 5-methylcytosine hydroxylases. Their requirement for $\alpha$-ketoglutarate, although likely accounting for only a small fraction of total $\alpha$-ketoglutarate utilization, makes this metabolite an essential component of cell signaling and epigenetic networks.

Conversion of glutamate to $\alpha$-ketoglutarate occurs either through oxidative deamination by glutamate dehydrogenase $(\mathrm{GDH})$ in the mitochondrion or by transamination to produce nonessential amino acids in either the cytosol or the mitochondrion. During avid glucose metabolism, the transamination pathway predominates (17). When glucose is scarce, GDH becomes a major pathway to supply glutamine carbon to the TCA cycle, and is required for cell survival $(17,18)$. Metabolism of glutaminederived $\alpha$-ketoglutarate in the TCA cycle serves several purposes: it generates reducing equivalents for the electron transport chain (ETC) and oxidative phosphorylation, becoming a major source 


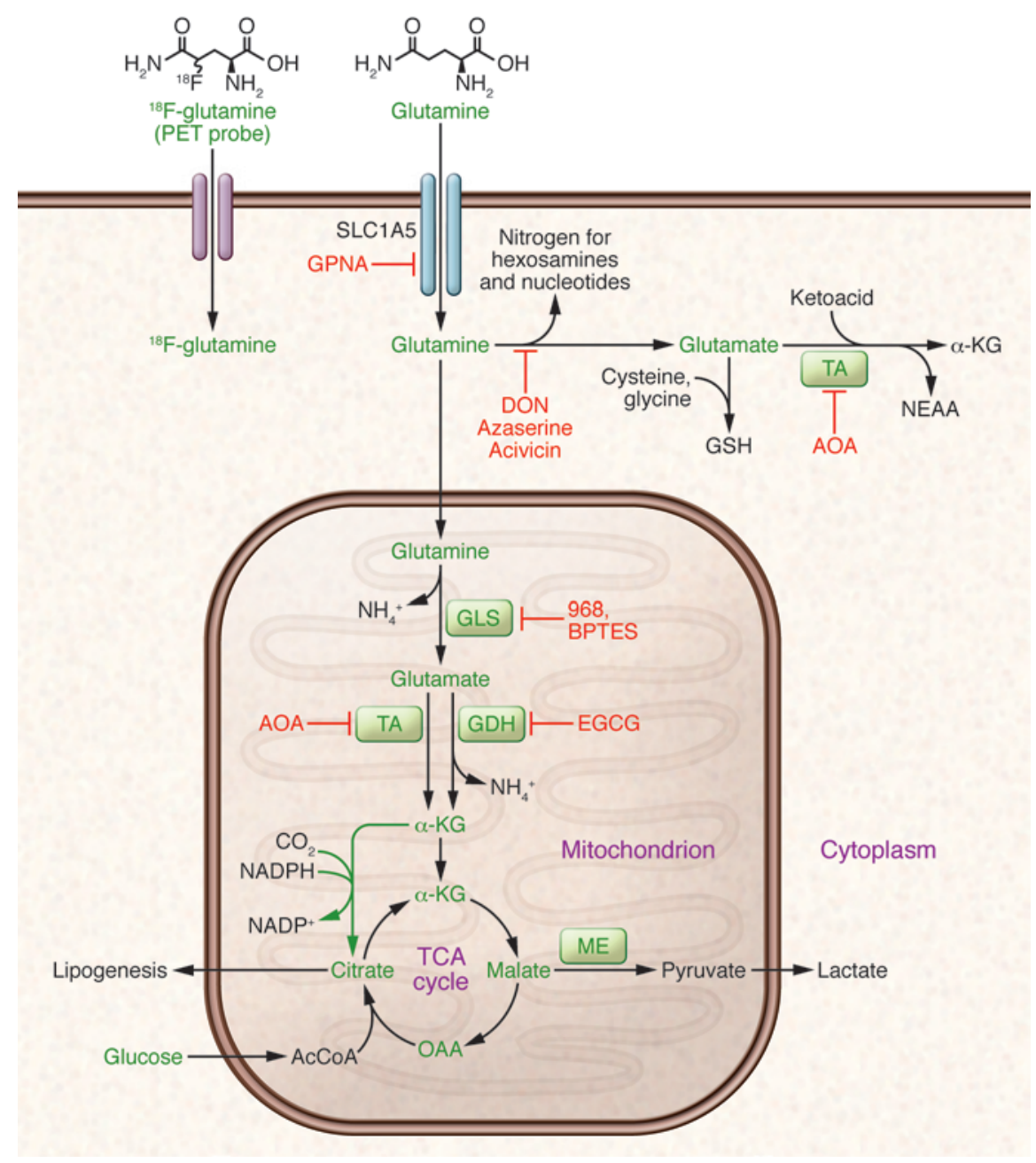

\section{Figure 1}

Glutamine metabolism as a target for diagnostic imaging and therapy in cancer. Glutamine is imported via SLC1A5 and other transporters, then enters a complex metabolic network by which its carbon and nitrogen are supplied to pathways that promote cell survival and growth. Enzymes discussed in the text are shown in green, and inhibitors that target various aspects of glutamine metabolism are shown in red. Green arrows denote reductive carboxylation. ${ }^{18} \mathrm{~F}$-labeled analogs of glutamine are also under development as PET probes for localization of tumor tissue. AcCoA, acetyl-CoA; DON, 6-diazo-5-oxo-L-norleucine; GSH, glutathione; NEAA, nonessential amino acids; ME, malic enzyme; OAA, oxaloacetate; TA, transaminase; 968, compound 968; $\alpha-K G$, $\alpha$-ketoglutarate. of energy (19); and it is an important anaplerotic nutrient, feeding net production of oxaloacetate to offset export of intermediates from the cycle to supply anabolism (20). Glutamine oxidation also supports redox homeostasis by supplying carbon to malic enzyme, some isoforms of which produce NADPH (Figure 1). In KRASdriven pancreatic adenocarcinoma cells, a pathway involving glutamine-dependent NADPH production is essential for redox balance and growth (21). In these cells, glutamine is used to produce aspartate in the mitochondria. This aspartate is then trafficked to the cytosol, where it is deaminated to produce oxaloacetate and then malate, the substrate for malic enzyme.

Recent work has uncovered an unexpected role for glutamine in cells with reduced mitochondrial function. Despite glutamine's conventional role as a respiratory substrate, several studies demonstrated a persistence of glutamine dependence in cells with permanent mitochondrial dysfunction from mutations in the ETC or TCA cycle, or transient impairment secondary to hypoxia (22-25). Under these conditions, glutamine-derived $\alpha$-ketoglutarate is reductively carboxylated by NADPH-dependent isoforms of isocitrate dehydrogenase to produce isocitrate, citrate, and other TCA cycle intermediates (Figure 1). These conditions broaden glutamine's utility as a carbon source because it becomes not only a major source of oxaloacetate, but also generates acetyl-CoA in what amounts to a striking rewiring of TCA cycle metabolism.

\section{Glutamine promotes hallmarks of malignancy}

Deregulated energetics. One hallmark of cancer cells is aberrant bioenergetics (26). Glutamine's involvement in the pathways outlined above contributes to a phenotype conducive to energy formation, survival, and growth. In addition to its role in mitochondrial metabolism, glutamine also suppresses expression of thioredoxininteracting protein, a negative regulator of glucose uptake (27). Thus, glutamine contributes to both of the energy-forming pathways in cancer cells: oxidative phosphorylation and glycolysis. Glutamine also modulates hallmarks not traditionally thought to be metabolic, as outlined below. These interactions highlight the complex interplay between glutamine metabolism and many aspects of cell biology.

Sustaining proliferative signaling. Pathological cancer cell growth relies on maintenance of proliferative signaling pathways with increased autonomy relative to non-malignant cells. Several lines of evidence argue that glutamine reinforces activity of these pathways. In some cancer cells, excess glutamine is exported in exchange for leucine and other essential amino acids. This exchange facilitates activation of the serine/threonine kinase mTOR, a major positive regulator of cell growth (28). In addition, glutamine-derived nitrogen is a component of amino sugars, known as hexosamines, that are used to glycosylate growth factor receptors and promote their localization to the cell surface. 


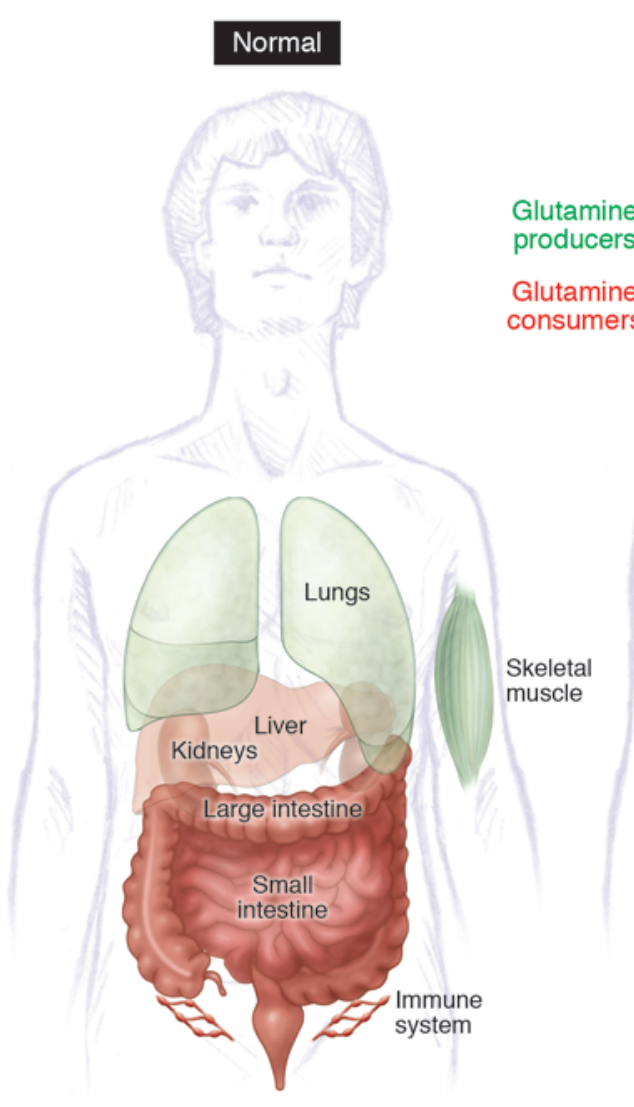

Disruption of hexosamine synthesis reduces the ability to initiate signaling pathways downstream of growth factors (29).

Enabling replicative immortality. Some aspects of glutamine metabolism oppose senescence and promote replicative immortality in cultured cells. In IMR90 lung fibroblasts, silencing either of two NADPH-generating isoforms of malic enzyme (ME1, ME2) rapidly induced senescence, while malic enzyme overexpression suppressed senescence (30). Both malic enzyme isoforms are repressed at the transcriptional level by p53 and contribute to enhanced levels of glutamine consumption and NADPH production in p53-deficient cells. The ability of p53-replete cells to resist senescence required the expression of ME1 and ME2, and silencing either enzyme reduced the growth of $\mathrm{TP} 3^{+/+}$and, to a lesser degree, TP53-1- tumors (30). These observations position malic enzymes as potential therapeutic targets.

Resisting cell death. Although many cancer cells require glutamine for survival, cells with enhanced expression of Myc oncoproteins are particularly sensitive to glutamine deprivation $(8,12,16)$. In these cells, glutamine deprivation induces depletion of TCA cycle intermediates, depression of ATP levels, delayed growth, diminished glutathione pools, and apoptosis. Myc drives glutamine uptake and catabolism by activating the expression of genes involved in glutamine metabolism, including GLS and SLC1A5, which encodes the $\mathrm{Na}^{+}$-dependent amino acid transporter ASCT2 $(12,16)$. Silencing GLS mimicked some of the effects of glutamine deprivation, including growth suppression in Myc-expressing cells and tumors $(10,12)$. MYCN amplification occurs in $20 \%-25 \%$ of neuroblastomas and is correlated with poor outcome (31). In cells with high $\mathrm{N}-\mathrm{Myc}$ levels, glutamine deprivation triggered an ATF4-dependent induction of apoptosis that could be prevented

\section{Figure 2}

Model for inter-organ glutamine metabolism in health and cancer. Organs that release glutamine into the bloodstream are shown in green, and those that consume glutamine are in red; the shade denotes magnitude of consumption/release. For some organs (liver, kidneys), evidence from model systems and/or human studies suggests that there is a change in net glutamine flux during tumorigenesis.

by restoring downstream metabolites oxaloacetate and $\alpha$-ketoglutarate (15). In this model, pharmacological activation of ATF4, inhibition of glutamine metabolic enzymes, or combinations of these treatments mimicked the effects of glutamine deprivation in cells and suppressed growth of MYCN-amplified subcutaneous and transgenic tumors in mice.

The PKC isoform PKC- $\zeta$ also regulates glutamine metabolism. Loss of PKC- $\zeta$ enhances glutamine utilization and enables cells to survive glucose deprivation (32). This effect requires flux of carbon and nitrogen from glutamine into serine. PKC- $\zeta$ reduces the expression of phosphoglycerate dehydrogenase, an enzyme required for glutamine-dependent serine biosynthesis, and also phosphorylates and inactivates this enzyme. Thus, PKC- $\zeta$ loss, which promotes intestinal tumorigenesis in mice, enables cells to alter glutamine metabolism in response to nutrient stress.

Invasion and metastasis. Loss of the epithelial cell-cell adhesion molecule E-cadherin is a component of the epithelial-mesenchymal transition, and is sufficient to induce migration, invasion, and tumor progression $(33,34)$. Addiction to glutamine may oppose this process because glutamine favors stabilization of tight junctions in some cells (35). Furthermore, the selection of breast cancer cells with the ability to grow without glutamine yielded highly adaptable subpopulations with enhanced mesenchymal marker expression and improved capacity for anchorage-independent growth, therapeutic resistance, and metastasis in vivo (36). It is unknown whether this result reflects a primary role for glutamine in suppressing these markers of aggressiveness in breast cancer, or whether prolonged glutamine deprivation selects for cells with enhanced fitness across a number of phenotypes.

\section{Organ-specific glutamine metabolism in health and disease}

As a major player in carbon and nitrogen transport, glutamine metabolism displays complex inter-organ dynamics, with some organs functioning as net producers and others as consumers (Figure 2). Organ-specific glutamine metabolism has frequently been studied in humans and animal models by measuring the arteriovenous difference in plasma glutamine abundance. In healthy subjects, the plasma glutamine pool is largely the result of release from skeletal muscle (37-39). In rats, the lungs are comparable to muscle in terms of glutamine production $(40,41)$, and human lungs also have the capacity for marked glutamine release, 


$\begin{array}{lllll} & & & \\ \text { Presentation } & \text { Prebiopsy diagnostics } & \text { Biopsy } & \text { Therapy } \\ \text { 2-year-old child } & \text { CT/MRI } & \text { Bone marrow biopsy } & \text { Chemotherapy } \\ \text { Failure to thrive } & \text { Urine VMA and HVA } & \text { Tumor biopsy } & \text { Radiation } & \text { CT/MRI } \\ \text { Abdominal mass } & \text { MIBG } & \text { Molecular pathology } & \text { Surgery } & \text { Mrine VMA and HVA } \\ & \text { Bone scan } & \text { for MYCN amplification } & \text { Glutamine } & \text { Bing } \\ & \text { Glutamine PET } & { }^{13} \text { C-glutamine infusion } & \text { metabolism inhibitors } & \text { Glutamine PET } \\ & & \text { of tumor } & \text { ATF4 agonists }\end{array}$

\section{Figure 3}

A strategy to integrate glutamine metabolism into the diagnosis, classification, treatment, and monitoring of neuroblastoma. Neuroblastoma commonly presents in children as an abdominal mass. A standard evaluation of a child with suspected neuroblastoma includes measurement of urine catecholamines, a bone scan, and full-body imaging with meta-iodobenzylguanidine (MIBG), all of which contribute to diagnosis and disease staging. In animal models, a subset of these tumors requires glutamine metabolism. This finding implies that approaches to image, quantify, or block glutamine metabolism (highlighted in red) in human neuroblastoma could be incorporated into the diagnosis and management of this disease. In particular, glutamine metabolic studies may help predict which tumors would respond to therapies targeting glutamine metabolism. HVA, homovanillic acid; VMA, vanillylmandelic acid.

although such release is most prominent in times of stress (42, 43). Stress-induced release from the lung is regulated by an induction of glutamine synthase expression as a consequence of glucocorticoid signaling and other mechanisms $(44,45)$. Although this results in a small arteriovenous difference, the overall release of glutamine is significant because of the large pulmonary perfusion. In rats and humans, adipose tissue is a minor but potentially important source of glutamine $(46,47)$. The liver has the capacity to synthesize or catabolize glutamine, with these activities subject both to regional heterogeneity among hepatocytes and regulatory effects of systemic acidosis and hyperammonemia. However, the liver does not appear to be a major contributor to the plasma glutamine pool in healthy rats and humans $(39,48,49)$.

Glutamine consumption occurs largely in the gut and kidney. The organs of the gastrointestinal tract drained by the portal vein, particularly the small intestine, are major consumers of plasma glutamine in both rats and humans $(37,38,49,50)$. Enterocytes oxidize more than half of glutamine carbon to $\mathrm{CO}_{2}$, accounting for a third of the respiration of these cells in fasting animals (51). The kidney consumes net quantities of glutamine to maintain acid-base balance $(37,38,52,53)$. During acidosis, the kidneys substantially increase their uptake of glutamine, cleaving it by GLS to produce ammonia, which is excreted along with organic acids to maintain physiologic $\mathrm{pH}(52,54)$. Glutamine is also a major metabolic substrate in lymphocytes and macrophages, at least during mitogenic stimulation of primary cells in culture (55-57).

Importantly, cancer seems to cause major changes in inter-organ glutamine trafficking (Figure 2). Currently, much work in this area is derived from studies in methylcholanthrene-induced fibrosarcoma in the rat, a model of an aggressively growing, glutamineconsuming tumor. In this model, fibrosarcoma induces skeletal muscle expression of glutamine synthetase and greatly increases the release of glutamine into the circulation. As the tumor increases in size, intramuscular glutamine pools are depleted in association with loss of lean muscle mass, mimicking the cachectic phenotype of humans in advanced stages of cancer (52). Simultaneously, both the liver and the kidneys become net glutamine exporters, although the hepatic effect may be diminished as the tumor size becomes very large $(48,49,52)$. Glutamine utilization by organs supplied by the portal vein is diminished in cancer (48). In addition to its function as a nutrient for the tumor itself, and possibly for cancer-associated immune cells, glutamine provides additional, indirect metabolic benefits to both the tumor and the host. For example, glutamine was used as a gluconeogenic substrate in cachectic mice with large orthotopic gliomas, providing a significant source of carbon in the plasma glucose pool (58). This glucose was taken up and metabolized by the tumor to produce lactate and to supply the TCA cycle.

It will be valuable to extend work in human inter-organ glutamine trafficking, both in healthy subjects and in cancer patients. Such studies will likely produce a better understanding of the pathophysiology of cancer cachexia, a major source of morbidity and mortality. Research in this area should also aid in the anticipation of organ-specific toxicities of drugs designed to interfere with glutamine metabolism. Alterations of glutamine handling in cancer may induce a different spectrum of toxicities compared with healthy subjects.

\section{Tumors differ according to their need for glutamine}

One important consideration is that not all cancer cells need an exogenous supply of glutamine. A panel of lung cancer cell lines displayed significant variability in their response to glutamine deprivation, with some cells possessing almost complete independence (59). Breast cancer cells also demonstrate systematic differences in glutamine dependence, with basal-type cells tending to be glutamine dependent and luminal-type cells tending to be glutamine independent (60). Resistance to glutamine deprivation is associated with the ability to synthesize glutamine de novo and/or to engage alternative pathways of anaplerosis $(10,60)$.

Tumors also display variable levels of glutamine metabolism in vivo. A study of orthotopic gliomas revealed that genetically diverse, human-derived tumors took up glutamine in the mouse brain but did not catabolize it (58). Rather, the tumors synthesized glutamine de novo and used pyruvate carboxylation for anaplerosis. Cells derived from these tumors did not require glutamine to survive or proliferate when cultured ex vivo. Glutamine synthesis from glucose was also a prominent feature of primary gliomas in human subjects infused with ${ }^{13} \mathrm{C}$-glucose at the time of surgical 
resection (61). Furthermore, an analysis of glutamine metabolism in lung and liver tumors revealed that both the tissue of origin and the oncogene influence whether the tumor produces or consumes glutamine (62). MET-induced hepatic tumors produced glutamine, whereas Myc-induced liver tumors catabolized it. In the lung, however, Myc expression was associated with glutamine accumulation.

This variability makes it imperative to develop ways to predict which tumors have the highest likelihood of responding to inhibitors of glutamine metabolism. Methods to image or otherwise quantify glutamine metabolism in vivo would be useful in this regard (63). Infusions of pre-surgical subjects with isotopically labeled glutamine, followed by extraction of metabolites from the tumor and analysis of ${ }^{13} \mathrm{C}$ enrichment, can be used to detect both glutamine uptake and catabolism $(58,62)$. However, this approach requires a specimen of the tumor to be obtained. Approaches for glutamine-based imaging, which avoid this problem, include a number of glutamine analogs compatible with PET. Although glutamine could in principle be imaged using the radioisotopes ${ }^{11} \mathrm{C},{ }^{13} \mathrm{~N}$, or ${ }^{18} \mathrm{~F}$, the relatively long half-life of the latter increases its appeal. In mice, ${ }^{18} \mathrm{~F}-(2 S, 4 R) 4$-fluoroglutamine is avidly taken up by tumors derived from highly glutaminolytic cells, and by glutamine-consuming organs including the intestine, kidney, liver, and pancreas (64). Labeled analogs of glutamate are also taken up by some tumors $(65,66)$. One of these, $(4 S)-4-\left(3-\left[{ }^{18} \mathrm{~F}\right]\right.$ fluoropropyl)L-glutamate $\left({ }^{18} \mathrm{~F}-\mathrm{FSPG}\right.$, also called BAY 94-9392), was evaluated in small clinical trials involving patients with several types of cancer $(65,67)$. This analog enters the cell through the cystine/glutamate exchange transporter ( $\mathrm{xC}^{-}$transport system), which is linked to glutathione biosynthesis (68). The analog was well tolerated, with high tumor detection rates and good tumor-to-background ratios in hepatocellular carcinoma and lung cancer.

PET approaches detect analog uptake and retention but cannot provide information about downstream metabolism. Analysis of hyperpolarized nuclei can provide a real-time view of enzymecatalyzed reactions. This technique involves redistribution of the populations of energy levels of a nucleus (e.g., $\left.{ }^{13} \mathrm{C},{ }^{15} \mathrm{~N}\right)$, resulting in a gain in magnetic resonance signal that can temporarily exceed 10,000-fold (69). This gain in signal enables rapid detection of both the labeled molecule and its downstream metabolites. Glutamine has been hyperpolarized on ${ }^{15} \mathrm{~N}$ and ${ }^{13} \mathrm{C}(70,71)$. In the latter case, the conversion of hyperpolarized glutamine to glutamate could be detected in intact hepatoma cells (70). If these analogs are translated to clinical studies, they might provide a dynamic view of the proximal reactions of glutaminolysis in vivo.

\section{Pharmacological strategies to inhibit glutamine metabolism in cancer}

Efforts to inhibit glutamine metabolism using amino acid analogs have an extensive history, including evaluation in clinical trials. Acivicin, 6-diazo-5-oxo-L-norleucine, and azaserine, three of the most widely studied analogs (Figure 1), all demonstrated variable degrees of gastrointestinal toxicity, myelosuppression, and neurotoxicity (72). Because these agents non-selectively target glutamine-consuming processes, recent interest has focused on developing methods directed at specific nodes of glutamine metabolism. First, ASCT2, the $\mathrm{Na}^{+}$-dependent neutral amino acid transporter encoded by SLC1A5, is broadly expressed in lung cancer cell lines and accounts for a majority of glutamine transport in those cells (Figure 1). It has been shown that $\gamma$-L-glutamylp-nitroanilide (GPNA) inhibits this transporter and limits lung cancer cell growth (73). Additional interest in GPNA lies in its ability to enhance the uptake of drugs imported via the monocarboxylate transporter MCT1. Suppressing glutamine uptake with GPNA enhances MCT1 stability and stimulates uptake of the glycolytic inhibitor 3-bromopyruvate (3-BrPyr) $(74,75)$. Because enforced MCT1 overexpression is sufficient to sensitize tumor xenografts to 3-BrPyr (76), GPNA may have a place in 3-BrPyrbased therapeutic regimens.

Two inhibitors of GLS isoforms have been characterized in recent years (Figure 1). Compound 968, an inhibitor of the GLSencoded splice isoform GAC, inhibits the transformation of fibroblasts by oncogenic RhoGTPases and delays the growth of GLS-expressing lymphoma xenografts (13). Bis-2-(5-phenylacetamido-1,2,4-thiadiazol-2-yl)ethyl sulfide (BPTES) also potently inhibits GLS isoforms encoded by GLS (77). BPTES impairs ATP levels and growth rates of P493 lymphoma cells under both normoxic and hypoxic conditions and suppresses the growth of P493-derived xenografts (78).

Evidence also supports a role for targeting the flux from glutamate to $\alpha$-ketoglutarate, although no potent, specific inhibitors yet exist to inhibit these enzymes in intact cells. Aminooxyacetate (AOA) inhibits aminotransferases non-specifically, but milliomolar doses are typically used to achieve this effect in cultured cells (Figure 1). Nevertheless, AOA has demonstrated efficacy in both breast adenocarcinoma xenografts and autochthonous neuroblastomas in mice $(15,79)$. Epigallocatechin gallate (EGCG), a green tea polyphenol, has numerous pharmacological effects, one of which is to inhibit GDH (80). The effects of EGCG on GDH have been used to kill glutamine-addicted cancer cells during glucose deprivation or glycolytic inhibition $(17,18)$ and to suppress growth of neuroblastoma xenografts (15).

\section{A paradigm to exploit glutamine metabolism in cancer}

Recent advances in glutamine-based imaging, coupled with the successful application of glutamine metabolic inhibitors in mouse models of cancer, make it possible to conceive of treatment plans that feature consideration of tumor glutamine utilization. A key challenge will be predicting which tumors are most likely to respond to inhibitors of glutamine metabolism. Neuroblastoma is used here as an example of a tumor in which evidence supports the utility of strategies that would involve both glutamine-based imaging and therapy (Figure 3). Neuroblastoma is the second most common extracranial solid malignancy of childhood. Highrisk neuroblastoma is defined by age, stage, and biological features of the tumor, including MYCN amplification, which occurs in some $20 \%-25 \%$ of cases (31). Because $M Y C N$-amplified tumor cells require glutamine catabolism for survival and growth (15), glutamine-based PET at the time of standard diagnostic imaging could help predict which tumors would be likely to respond to inhibitors of glutamine metabolism. Infusion of ${ }^{13} \mathrm{C}$-glutamine coordinated with the diagnostic biopsy could then enable inspection of ${ }^{13} \mathrm{C}$ enrichment in glutamine-derived metabolites from the tumor, confirming the activity of glutamine catabolic pathways. Following on evidence from mouse models of neuroblastoma, treatment could then include agents directed against glutamine catabolism (15). Of note, some tumors were sensitive to the ATF4 agonist fenretinide (FRT), alone or in combination with EGCG. Importantly, FRT has already been the focus of a Phase I clinical trial in children with solid tumors, including neuroblastoma, and was fairly well tolerated (81). 


\section{Conclusions}

Glutamine is a versatile nutrient required for the survival and growth of a potentially large subset of tumors. Work over the next several years should produce a more accurate picture of the molecular determinants of glutamine addiction and the identification of death pathways that execute cells when glutamine catabolism is impaired. Advancement of glutamine-based imaging into clinical practice should soon make it possible to differentiate tumors that take up glutamine from those that do not. Finally, the development of safe, high-potency inhibitors of key metabolic nodes should facilitate therapeutic regimens featuring inhibition of glutamine metabolism.

1. Schulze A, Harris AL. How cancer metabolism is tuned for proliferation and vulnerable to disruption. Nature. 2012;491(7424):364-373

2. Dang CV. Links between metabolism and cancer. Genes Dev. 2012;26(9):877-890.

3. Mullen AR, DeBerardinis RJ. Genetically-defined metabolic reprogramming in cancer. Trends Endocrinol Metab. 2012;23(11):552-559.

4. DeBerardinis RJ, Cheng T. Q's next: the diverse functions of glutamine in metabolism, cell biology and cancer. Oncogene. 2009;29(3):313-324.

5. Medina MA, Sanchez-Jimenez F, Marquez J, Rodriguez Quesada A, Nunez de Castro I. Relevance of glutamine metabolism to tumor cell growth. $\mathrm{Mol}$ Cell Biochem. 1992;113(1):1-15.

6. Bergström J, Fürst P, Norée LO, Vinnars E. Intracellular free amino acid concentration in human muscle tissue. J Appl Physiol. 1974;36(6):693-697.

7. Lacey JM, Wilmore DW. Is glutamine a conditionally essential amino acid? Nutr Rev. 1990;48(8):297-309.

8. Yuneva M, Zamboni N, Oefner P, Sachidanandam $\mathrm{R}$, Lazebnik Y. Deficiency in glutamine but not glucose induces MYC-dependent apoptosis in human cells. J Cell Biol. 2007;178(1):93-105.

9. Matés JM, Segura JA, Martín-Rufián M, CamposSandoval JA, Alonso FJ, Márquez J. Glutaminase isoenzymes as key regulators in metabolic and oxidative stress against cancer. Curr Mol Med. 2012; 13(4):514-534.

10. Cheng T, et al. Pyruvate carboxylase is required for glutamine-independent growth of tumor cells. Proc Natl Acad Sci U S A. 2011;108(21):8674-8679.

11. Lobo C, Ruiz-Bellido MA, Aledo JC, Marquez J, Nunez De Castro I, Alonso FJ. Inhibition of glutaminase expression by antisense mRNA decreases growth and tumourigenicity of tumour cells. Biochem J. 2000;348(pt 2):257-261.

12. Gao P, et al. c-Myc suppression of miR-23a/b enhances mitochondrial glutaminase expression and glutamine metabolism. Nature. 2009;458(7239):762-765.

13. Wang JB, et al. Targeting mitochondrial glutaminase activity inhibits oncogenic transformation. Cancer Cell. 2010;18(3):207-219.

14. Suzuki S, et al. Phosphate-activated glutaminase (GLS2), a p53-inducible regulator of glutamine metabolism and reactive oxygen species. Proc Natl Acad Sci U S A. 2010;107(16):7461-7466.

15. Qing G, et al. ATF4 regulates MYC-mediated neuroblastoma cell death upon glutamine deprivation. Cancer Cell. 2012;22(5):631-644.

16 . Wise DR, et al. Myc regulates a transcriptional program that stimulates mitochondrial glutaminolysis and leads to glutamine addiction. Proc Natl Acad SciU S A. 2008;105(48):18782-18787.

17. Yang C, Sudderth J, Dang T, Bachoo RG, McDonald JG, DeBerardinis RJ. Glioblastoma cells require glutamate dehydrogenase to survive impairments of glucose metabolism or Akt signaling. Cancer Res. 2009;69(20):7986-7993.

18. Choo AY, et al. Glucose addiction of TSC null cells is caused by failed mTORC1-dependent balanc-

\section{Acknowledgments}

We thank members of the DeBerardinis laboratory for critically reading the manuscript, and Angela Diehl for expert assistance with the figures. C.T. Hensley is supported by an NIH training grant (5T32GM007062-38), A.T. Wasti is supported by the Children's Cancer Fund, and R.J. DeBerardinis is supported by grants from the NIH (1R01CA157996-02), Damon-Runyon Cancer Research Foundation, and Welch Foundation (I-1733).

Address correspondence to: Ralph J. DeBerardinis, 5323 Harry Hines Blvd., Room NL12.138B, Dallas, Texas 75390-8502, USA. Phone: 214.633.1804; Fax: 214.648.5402; E-mail: Ralph. deberardinis@utsouthwestern.edu. ing of metabolic demand with supply. Mol Cell. 2010;38(4):487-499.

19. Reitzer LJ, Wice BM, Kennell D. Evidence that glutamine, not sugar, is the major energy source for cultured HeLa cells. J Biol Chem. 1979;254(8):2669-2676.

20. DeBerardinis RJ, et al. Beyond aerobic glycolysis: Transformed cells can engage in glutamine metabolism that exceeds the requirement for protein and nucleotide synthesis. Proc Natl Acad Sci U S A. 2007; 104(49):19345-19350.

21. Son J, et al. Glutamine supports pancreatic cancer growth through a KRAS-regulated metabolic pathway. Nature. 2013;496(7443):101-105.

22. Metallo CM, et al. Reductive glutamine metabolism by IDH1 mediates lipogenesis under hypoxia. Nature. 2012;481(7381):380-384.

23. Wise DR, et al. Hypoxia promotes isocitrate dehydrogenase-dependent carboxylation of alpha-ketoglutarate to citrate to support cell growth and viability. Proc Natl Acad Sci U S A. 2011;108(49):19611-19616.

24. Scott DA, et al. Comparative metabolic flux profiling of melanoma cell lines: beyond the Warburg effect. J Biol Chem. 2011;286(49):42626-42634.

25. Mullen AR, et al. Reductive carboxylation supports growth in tumour cells with defective mitochondria. Nature. 2011;481(7381):385-388.

26. Hanahan D. Hallmarks of cancer: the next generation. Cell. 2011;144(5):646-674

27. Kaadige MR, Looper RE, Kamalanaadhan S, Ayer DE. Glutamine-dependent anapleurosis dictates glucose uptake and cell growth by regulating MondoA transcriptional activity. Proc Natl Acad SciU S A. 2009;106(35):14878-14883.

28. Nicklin P, et al. Bidirectional transport of amino acids regulates mTOR and autophagy. Cell. 2009; 136(3):521-534.

29. Wellen KE, et al. The hexosamine biosynthetic pathway couples growth factor-induced glutamine uptake to glucose metabolism. Genes Dev. 2010;24(24):2784-2799.

30. Jiang P, Du W, Mancuso A, Wellen KE, Yang X. Reciprocal regulation of p53 and malic enzymes modulates metabolism and senescence. Nature. 2013;493(7434):689-693.

31. Maris JM. Recent advances in neuroblastoma. N Engl J Med. 2010;362(23):2202-2211.

32. Ma L, et al. Control of nutrient stress-induced metabolic reprogramming by $\mathrm{PKC} \zeta$ in tumorigenesis. Cell. 2013;152(3):599-611.

33. Cavallaro U, Christofori G. Cell adhesion and signalling by cadherins and Ig-CAMs in cancer. Nat Rev Cancer. 2004;4(2):118-132.

34. Perl AK, Wilgenbus P, Dahl U, Semb H, Christofori $G$. A causal role for E-cadherin in the transition from adenoma to carcinoma. Nature. 1998; 392(6672):190-193.

35. Seth A, Basuroy S, Sheth P, Rao RK. L-Glutamine ameliorates acetaldehyde-induced increase in paracellular permeability in Caco- 2 cell monolayer. Am J Physiol Gastrointest Liver Physiol. 2004;287(3):G510-G517.

36. Singh B, et al. Selection of metastatic breast cancer cells based on adaptability of their metabolic state. PLoS One. 2012;7(5):e36510.

37. Stumvoll M, Perriello G, Meyer C, Gerich J. Role of glutamine in human carbohydrate metabolism in kidney and other tissues. Kidney Int. 1999; 55(3):778-792.

38. Lochs H, Roth E, Gasic S, Hübl W, Morse EL, Adibi SA. Splanchnic, renal, and muscle clearance of alanylglutamine in man and organ fluxes of alanine and glutamine when infused in free and peptide forms. Metabolism. 1990;39(8):833-836.

39. Felig P, Wahren J, Räf L. Evidence of inter-organ amino-acid transport by blood cells in humans. Proc Natl Acad Sci U S A. 1973;70(6):1775-1779.

40. Plumley DA, Austgen TR, Salloum RM, Souba WW. Role of the lungs in maintaining amino acid homeostasis. JPEN J Parenter Enteral Nutr. 1990;14(6):569-573.

41. Souba WW, Herskowitz K, Plumley DA. Lung glutamine metabolism. JPEN J Parenter Enteral Nutr. 1990;14(4 suppl):68S-70S.

42. Plumley DA, et al. Accelerated lung amino acid release in hyperdynamic septic surgical patients. Arch Surg. 1990;125(1):57-61.

43. Hulsewé KE, Hulst RWJ, Ramsay G, Berlo CH, Deutz NP, Soeters P. Pulmonary glutamine production: effects of sepsis and pulmonary infiltrates. Intensive Care Med. 2003;29(10):1833-1836.

44. Pan M, Wasa M, Ryan U, Souba W. Inhibition of pulmonary microvascular endothelial glutamine transport by glucocorticoids and endotoxin. JPEN J Parenter Enteral Nutr. 1995;19(6):477-481.

45. Labow BI, Abcouwer SF, Lin C-M, Souba WW. Glutamine synthetase expression in rat lung is regulated by protein stability. Am J Physiol. 1998; 275(5 pt 1):L877-L886.

46. Patterson BW, Horowitz JF, Wu G, Watford M, Coppack SW, Klein S. Regional muscle and adipose tissue amino acid metabolism in lean and obese women. $A m$ J Physiol Endocrinol Metab. 2002;282(4):E931-E936.

47. Tischler ME, Goldberg AL. Leucine degradation and release of glutamine and alanine by adipose tissue. J Biol Chem. 1980;255(17):8074-8081.

48. Souba WW, Strebel FR, Bull JM, Copeland EM, Teagtmeyer $\mathrm{H}$, Cleary K. Interorgan glutamine metabolism in the tumor-bearing rat.J Surg Res. 1988;44(6):720-726.

49. Dudrick PS, Inoue Y, Espat NJ, Souba WW. Na+dependent glutamine transport in the liver of tumour-bearing rats. Surg Oncol. 1993;2(3):205-215.

50. Souba WW, Herskowitz K, Salloum RM, Chen MK, Austgen TR. Gut glutamine metabolism. JPENJ Parenter Enteral Nutr. 1990;14(4 suppl):45S-50S.

51. Windmueller HG. Glutamine utilization by the small intestine. Adv Enzymol Relat Areas Mol Biol. 1982;53:201-237.

52. Chen MK, Espat NJ, Bland KI, Copeland EM, Souba WW. Influence of progressive tumor growth on glutamine metabolism in skeletal muscle and kidney. Ann Surg. 1993;217(6):655-667.

53. Brundin T, Wahren J. Renal oxygen consumption, 
thermogenesis, and amino acid utilization during i.v. infusion of amino acids in man. Am J Physiol. 1994;267(5 pt 1):E648-E655.

54. Taylor L, Curthoys NP. Glutamine metabolism: role in acid-base balance* Biochem Mol Biol Educ. 2004;32(5):291-304.

55. Ardawi MS, Newsholme EA. Glutamine metabolism in lymphocytes of the rat. Biochem J. 1983; 212(3):835-842.

56. Ardawi MS. Glutamine and glucose metabolism in human peripheral lymphocytes. Metabolism. 1988; 37(1):99-103.

57. Newsholme P, Newsholme EA. Rates of utilization of glucose, glutamine and oleate and formation of end-products by mouse peritoneal macrophages in culture. Biochem J. 1989;261(1):211-218.

58. Marin-Valencia I, et al. Analysis of tumor metabolism reveals mitochondrial glucose oxidation in genetically diverse human glioblastomas in the mouse brain in vivo. Cell Metab. 2012;15(6):827-837.

59. van den Heuvel AP, Jing J, Wooster RF, Bachman KE. Analysis of glutamine dependency in nonsmall cell lung cancer: GLS1 splice variant GAC is essential for cancer cell growth. Cancer Biol Ther. 2012;13(12):1185-1194.

60. Kung HN, Marks JR, Chi JT. Glutamine synthetase is a genetic determinant of cell type-specific glutamine independence in breast epithelia. PLoS Genet. 2011;7(8):e1002229.

61. Maher EA, et al. Metabolism of [U-13C]glucose in human brain tumors in vivo. NMR Biomed. 2012; 25(11):1234-1244.

62. Yuneva MO, et al. The metabolic profile of tumors depends on both the responsible genetic lesion and tissue type. Cell Metab. 2012;15(2):157-170.
63. Rajagopalan KN, DeBerardinis RJ. Role of glutamine in cancer: therapeutic and imaging implications. J Nucl Med. 2011;52(7):1005-1008.

64. Lieberman BP, et al. PET imaging of glutaminolysis in tumors by $18 \mathrm{~F}-(2 \mathrm{~S}, 4 \mathrm{R}) 4$-fluoroglutamine. J Nucl Med. 2011;52(12):1947-1955.

65. Baek S, et al. Exploratory clinical trial of (4S)-4(3- $\left[{ }^{18} \mathrm{~F}\right]$ fluoropropyl)-L-glutamate for imaging $\mathrm{x}_{\mathrm{C}}{ }^{-}$ transporter using positron emission tomography in patients with non-small cell lung or breast cancer. Clin Cancer Res. 2012;18(19):5427-5437.

66. Ploessl K, Wang L, Lieberman BP, Qu W, Kung HF. Comparative evaluation of $18 \mathrm{~F}$-labeled glutamic acid and glutamine as tumor metabolic imaging agents. J Nucl Med. 2012;53(10):1616-1624.

67. Baek S, et al. (4S)-4-(3-18F-fluoropropyl)-L-glutamate for imaging of $\mathrm{xC}$ transporter activity in hepatocellular carcinoma using PET: preclinical and exploratory clinical studies. J Nucl Med. 2013;54(1):117-123.

68. Koglin $N$, et al. Specific PET imaging of $\mathrm{x}_{\mathrm{C}^{-}}$trans porter activity using a ${ }^{18} \mathrm{~F}$-labeled glutamate derivative reveals a dominant pathway in tumor metabolism. Clin Cancer Res. 2011;17(18):6000-6011.

69. Kurhanewicz J, et al. Analysis of cancer metabolism by imaging hyperpolarized nuclei: prospects for translation to clinical research. Neoplasia. 2011;13(2):81-97.

70. Gallagher FA, Kettunen MI, Day SE, Lerche M, Brindle KM. 13C MR spectroscopy measurements of glutaminase activity in human hepatocellular carcinoma cells using hyperpolarized 13C-labeled glutamine. Magn Reson Med. 2008;60(2):253-257.

71. Barb AW, Hekmatyar SK, Glushka JN, Prestegard $\mathrm{JH}$. Exchange facilitated indirect detection of hyperpolarized 15ND2-amido-glutamine. J Magn
Reson. 2011;212(2):304-310

72. Ahluwalia GS, Grem JL, Hao Z, Cooney DA. Metabolism and action of amino acid analog anti-cancer agents. Pharmacol Ther. 1990;46(2):243-271.

73. Hassanein M, et al. SLC1A5 mediates glutamine transport required for lung cancer cell growth and survival. Clin Cancer Res. 2013;19(3):560-570.

74. Cardaci S, Ciriolo MR. Deprive to kill: Glutamine closes the gate to anticancer monocarboxylic drugs. Autophagy. 2012;8(12):1830-1832.

75. Cardaci S, et al. Glutamine deprivation enhances antitumor activity of 3-bromopyruvate through the stabilization of monocarboxylate transporter-1. Cancer Res. 2012;72(17):4526-4536.

76. Birsoy K, et al. MCT1-mediated transport of a toxic molecule is an effective strategy for targeting glycolytic tumors. Nat Genet. 2013;45(1):104-108.

77. Robinson MM, et al. Novel mechanism of inhibition of rat kidney-type glutaminase by bis-2-(5phenylacetamido-1,2,4-thiadiazol-2-yl)ethyl sulfide (BPTES). Biochem J. 2007;406(3):407-414.

78. Le A, et al. Glucose-independent glutamine metabolism via TCA cycling for proliferation and survival in B cells. Cell Metab. 2012;15(1):110-121.

79. Thornburg JM, et al. Targeting aspartate aminotransferase in breast cancer. Breast Cancer Res. 2008; 10(5):R84.

80. Li C, et al. Green tea polyphenols modulate insulin secretion by inhibiting glutamate dehydrogenase. J Biol Chem. 2006;281(15):10214-10221.

81. Villablanca JG, Krailo MD, Ames MM, Reid JM, Reaman GH, Reynolds CP. Phase I trial of oral fenretinide in children with high-risk solid tumors: a report from the Children's Oncology Group (CCG 09709). J Clin Oncol. 2006;24(21):3423-3430. 